\title{
The Influence of Dopamine Receptor D4 Polymorphism on Resting EEG in Healthy Young Females
}

\author{
Tien-Wen Lee ${ }^{1}$, Younger W.-Y. Yu ${ }^{2}$, Chen-Jee Hong ${ }^{3,4}$, Shih-Jen Tsai ${ }^{3,4}$, Hung-Chi $\mathrm{Wu}^{5}$ and \\ Tai-Jui Chen ${ }^{*}, 6$
}

\author{
${ }^{1}$ Laureate Institute for Brain Research, Tulsa, OK 74136, USA \\ ${ }^{2} Y u$ 's Psychiatric Clinic, Kaohsiung, Taiwan \\ ${ }^{3}$ Department of Psychiatry, Taipei Veterans General Hospital, Taipei, Taiwan \\ ${ }^{4}$ School of Medicine, National Yang-Ming University, Taipei, Taiwan \\ ${ }^{5}$ Kai-Suan Psychiatric Hospital, Kaohsiung, Taiwan \\ ${ }^{6}$ Department of Psychiatry, E-DA Hospital, Kaohsiung County, Taiwan
}

\begin{abstract}
The polymorphism of variable number of tandem repeat (VNTR) in dopamine receptor D4 (DRD4) gene exon III has been linked to various neuro-psychiatric conditions with disinhibition/impulsivity as one of the core features. This study examined the modulatory effects of long-allele variant of DRD4 VNTR on the regional neural activity as well as inter-regional neural interactions in a young female population. Blood sample and resting state eyes-closed EEG signals were collected in 233 healthy females, stratified into two groups by polymerase chain reaction: long-allele carriers ( $>4-$ repeat) and non-carriers ( $<=4$-repeat $/<=4$-repeat). The values of mean power of 18 electrodes and mutual information of 38 channel pairs across theta, alpha, and beta frequencies were analyzed. Our connectivity analysis was based on information theory, which combined Morlet wavelet transform and mutual information calculation. Between-group differences of regional power and connectivity strength were quantified by independent t-test, while between-group differences in global trends were examined by non-parametric analyses. We noticed that DRD4 VNTR long-allele was associated with decreased global connectivity strength (from non-parametric analysis), especially over bi-frontal, biparietal and right fronto-parietal and right fronto-temporal connections (from independent t-tests). The between-group differences in regional power were not robust. Our findings fit with the networks of response inhibition, providing evidence bridging DRD4 long-allele and disinhibition/impulsivity in neuropsychiatric disorders. We suggest future DRD4 studies of imaging genetics incorporate connectivity analysis to unveil its impact on cerebral network.
\end{abstract}

Keywords: Electroencephalography (EEG), mutual information, connectivity, power spectrum, DRD4, polymorphism.

\section{INTRODUCTION}

The D4 dopamine receptor (DRD4) is a member of the family of D2-like dopamine receptors, located on chromosome $11 \mathrm{p} 15.5$. D2-like receptor signaling is mediated by several classes of $\mathrm{G}$ proteins, which regulate the activities of adenylate cyclase, ion channels, phospholipases, protein kinases, and receptor tyrosine kinases, and is also modulated by other protein-protein interactions [1]. The messenger ribonucleic acids (mRNAs) of D2, D3, and D4 receptors are identifiable in the hippocampal formation and in the cortical regions of the medial temporal lobe. Unlike DRD2, DRD4 mRNAs are abundant in the prefrontal and temporal neocortex, while DRD2 mRNAs distribute homogenously throughout the striatal structures $[2,3]$. It was thus assumed that the DRD4 gene is likely to impact the functioning of the brain cortex in a direct way, whereas in contrast, the DRD2 gene might influence frontal cortex indirectly through fronto-striatal interaction. Although DRD4 has been largely

*Address correspondence to this author at the Department of Psychiatry, E-DA Hospital, Kaohsing County, Taiwan; Tel: +886-(0)7-6150011; Fax: +886-(0)7-6155352; E-mails: tjchen71@kimo.com; ed100239@edah.org.tw related to prefrontal function, an animal study suggested that the modulatory effect of DRD4 on cerebral metabolism was not restricted to prefrontal cortex, compatible with the observed mRNA distribution across several distinct brain regions [4].

A functional polymorphism of variable number of tandem repeat (VNTR) at DRD4 exon III, a 48-base-pair sequence in the genetic code of third cytoplasmic loop, was first discovered by Van Tol et al., who demonstrated different properties for the longer alleles (7 repeats) and the shorter alleles ( 2 or 4 repeats) with respect to clozapine and spiperone binding [5]. The potency of dopamine to inhibit cyclic AMP formation was about twofold reduced for 7 repeat allele compared with the 2-repeat and 4-repeat variants of DRD4 [6]. The 48-base-pair repeat number may range from 2 to 11 and the frequency of the alleles varies greatly between populations. Meta-analyses have suggested a significant association between the long-repeat (7- or 7and 5- repeat) polymorphism and attention deficit hyperactivity disorder (ADHD), while the 4-repeat allele has protective effects $[7,8]$. The DRD4 exon III VNTR polymorphism has also been linked to other neuro-psychiatric conditions 
such as Tourette syndrome, migraine, substance abuse, bulimia nervosa and major depression [9-14]. In normal population, the DRD4 VNTR polymorphism also differentiates neuro-psychological performance. It was suggested that the long-allele was associated with risk taking, slower reaction time, lower persistence and impulsivity [15-19]. The presence of a 7-repeat allele was also accompanied with inaccurate/impulsive response style in ADHD [20-22].

Concordant with the observation of the cortical distribution of mRNA and the possible roles in cortical functioning, electroencephalography (EEG) studies demonstrated that the DRD4 VNTR polymorphism modulated cortical electrical activities. Strobel et al., explored DRD4 exon III genotype, eye-blink rate (a measure of central dopaminergic neurotransmission) and novelty P300, and concluded that the DRD4 exon III polymorphism influences the processing of novelty, which was further modulated by tonic dopaminergic activity [23]. In addition, it was reported that the 7-repeat allele of the DRD4 polymorphism enhanced the auditory evoked responses to both standard and target stimuli [24]. In the Go/Nogo task, 7-repeat carriers were noticed to present an increased Nogo-related theta band response and a reduced go-related beta decrease, supporting the modulatory role of DRD4 in prefrontal areas related to inhibitory mechanism [25]. The approach of imaging genetics applying the tasks of response inhibition is of particular interest given that the decline in inhibitory control or impulsivity is a common feature of various neuro-psychiatric disorders relevant to DRD4 and that the DRD4 VNTR polymorphism has been recognized to exert impact on the capability of inhibiting pre-potent reaction $[18,19,26-28]$. It is intriguing to note that the relationship between inhibition/impulsivity and DRD4 may also account for, at least partly, previous studies that related DRD4 VNTR to lower persistence, risk taking and differences in reaction time [15-17].

This study planned to investigate the effect of DRD4 VNTR polymorphism on resting EEG, which has never been examined before. Resting EEG carries abundant information predictive of performance on several neuro-psychological tasks, and even the early stage of Alzheimer's disease or the treatment response of major depressive disorder [29-34]. We were particularly interested in the inferior frontal gyrus and right-lateralized network since the processing of response inhibition has been noticed to be dominant in right hemisphere [35-44]. Since dopamine system has been known to affect inter-regional interaction [45], we quantified both local power and inter-regional connectivity strength. To investigate the genotype-connectivity relationship, we adopted an information-theory-based approach developed by Chen et al., which may accommodate non-linear neural interaction [46]. Given that gender effect has been noticed for DRD4 exon III polymorphism on cognition and social behaviors, and the long-allele is relatively rare in HanChinese, we restricted this research sample to females to eschew potential gender interaction [47-50].

\section{MATERIALS AND METHODOLOGY}

\section{Subjects}

We enrolled 233 right-handed healthy young females, with their ages ranging from 19 to 21 years. The neurolo- gical and physical examinations were performed by licensed medical doctors. A semi-structural interview process was performed to evaluate their psychiatric condition by licensed psychiatrists. Those who had a history of major medical or neurological disorder, substance abuse or psychiatric disease were excluded. Only those who had been medication-free, including birth control pills, for at least two weeks were recruited. This project was approved by the local ethical committee, complying with the Helsinki Declaration. The informed consents were obtained from all participants prior to the commencement of the investigation.

\section{EEG Recordings and Analyses}

All participants received a 3-minute conventional, eyesclosed, awake, digital EEG after a 5-minute habituation to the experimental environment (Brain Atlas III computer, Biologic System Company, Chicago). Recordings followed the standard of international 10-20 system with ear-linked reference, at a $128 \mathrm{~Hz}$ sampling rate and impedance below 3 $\mathrm{k} \Omega$, high pass filter $0.05 \mathrm{~Hz}$, low pass filter $70 \mathrm{~Hz}$, notch filter $60 \mathrm{~Hz}$ [51]. The frequency bands were defined as follows: theta 4 to $8 \mathrm{~Hz}$, alpha 8 to $12 \mathrm{~Hz}$, beta 12 to $24 \mathrm{~Hz}$, beta1 12 to $18 \mathrm{~Hz}$ and beta 18 to $24 \mathrm{~Hz}$. The artifact of vertical eyeball movement was detected from electrodes placed above and below the right eye, with the horizontal analog derived from electrodes placed at the left outer canthus. EEG artifacts were handled by semi-automated module provided by software EEGLAB (http://sccn.ucsd.edu/eeglab). Various sources of artifact, such as external artifact, movements, oculogenic potentials and myogenic potentials, were detected and deleted via visual inspection by experienced EEG technician and then the signal quality was examined by channel statistics and QQ-plot. Twenty electrodes were used to acquire EEG data and all the EEG signals were re-referenced to the average activity of all the electrodes. The electrodes F7, F3, Fz, F4, F8, T3, C3, Cz, C4, T4, T5, P3, Pz, P4, T6, $\mathrm{O} 1, \mathrm{Oz}$ and $\mathrm{O} 2$ were included in the analyses. We made a systemic approach to examine the functional connectivity of 38 electrode pairs: (1) 7 symmetrical connections of F7-F8, F3-F4, C3-C4, T3-T4, T5-T6, P3-P4 and O1-O2; (2) 15 ipsilateral channel pairs of the left hemisphere from all the possible combinations of F3, F7, C3, P3, T3 and T5; (3) 15 ipsilateral channel pairs of the right hemisphere from all the possible combinations of F4, F8, C4, P4, T4 and T6; (4) 1 midline anterior-posterior connection of $\mathrm{Fz}-\mathrm{Pz}$.

Fast Fourier Transform (FFT) was used to derive the mean EEG power (unit: $\mu \mathrm{V}^{2}$ ), and a mutual-informationbased approach was adopted to investigate the functional connectivity strengths between coupled EEG channels, namely time-frequency cross mutual information (TFCMI) method [46], which has been applied in our previous EEGgenetic study [52]. As to the regional power analyses, FFT was applied to consecutively non-overlapped and artifactfree segments of $20 \mathrm{sec}$ to derive the mean EEG power for each electrode at a specified frequency band [53]. As to the connectivity analyses, TFCMI method first transformed the EEG time series into power series over pre-specified frequency bands by Morlet wavelet method. The dependency, i.e. functional connectivity, between the spectral dynamics at two different EEG channels was then calculated by mutual information (MI). 
To examine whether there was a global trend difference in the regional mean power and connectivity strength across different frequency bands between the 2 genotyped groups, we performed non-parametric analyses. Our null hypothesis assumed that the probability of a certain index (i.e., regional mean power or connectivity strength of coupled electrodes) at a specific frequency band, group one is greater than group two equals the probability that group two is greater than group one (i.e., the probability was 0.5 ). The probability to obtain $\mathrm{j}$ or more "group one $>$ group two" indices by chance can be calculated using the following formula:

$$
\mathrm{P}=\sum_{\mathrm{k}=\mathrm{j}}^{\mathrm{s}}(\mathrm{s}) \times 0.5^{\mathrm{s}}
$$

where $\mathrm{s}$ is the total number of comparisons. The nonparametric analyses were executed for regional power and inter-regional interaction respectively: [1] one comparison for each electrode-frequency couple e.g., $\mathrm{F} 3$ alpha; $\mathrm{s}=18 \times 5$ when taking all the electrode(18)-frequency(5) pairs into account, and [2] one comparison for each connection-frequency couple e.g., F7-F8 alpha; $s=37 \times 5$ when considering all the connection(37)-frequency(5) pairs.

\section{Genotyping of DRD4 Exon III VNTR Polymorphism}

Genomic DNA was extracted from peripheral blood leukocytes and was amplified by polymerase chain reaction (PCR) using the primers designed by Shaikh et al. [54]. The PCR ingredient and procedure were described as follows. The PCR reagent contained $50 \mathrm{mM} \mathrm{KCl}, 10 \mathrm{mM}$ tris- $\mathrm{HCl}$ at $\mathrm{pH} 8.3,0.5 \mathrm{mM} \mathrm{MgCl} 2,10 \%$ dimethylsulfoxide (DMSO), $200 \mathrm{mM}$ of dATP, dTTP, and dCTP, $50 \mathrm{mM}$ of dGTP, and $150 \mathrm{mM}$ of 7-deaza-guanosine, $0.5 \mathrm{mM}$ of each primer, 100 ng template DNA, and 0.6 units of Dynazyme. The mixture was denatured at $95^{\circ} \mathrm{C}$ for $5 \mathrm{~min}$, followed by 30 cycles of amplification $\left(94^{\circ} \mathrm{C}, 1 \mathrm{~min} ; 52^{\circ} \mathrm{C}, 1 \mathrm{~min} ; 72^{\circ} \mathrm{C}, 2 \mathrm{~min}\right)$ and 5 min of elongation at $72^{\circ} \mathrm{C}$. Amplified DRD4 fragments were detected by $2 \%$ ethidium bromide-stained agarose gel electrophoresis, with the genotype determined by size fractionation under ultraviolet illumination [55].

\section{Statistical Analyses}

The participants were categorized into 2 groups according to the allele length of DRD4 VNTR. We lumped the participants with both alleles no longer than 4-repeat $(<=4 /<=4)$ as group 1 and those with at least one allele longer than 4-repeat as group 2. Independent t-test with assumed unequal variance was performed to elucidate the electrodes and channel-frequency pairs with values of mean power and mutual information showing significant betweengroup differences, respectively. For each test set in this study, the criterion for significance was set at $P<0.05$, twotailed. We assumed the independency of each frequency band and performed the Bonferroni correction based on $P=$ $1-(1-0.05)^{1 / n}$, where $\mathrm{n}$ equals the number of comparisons, with $\mathrm{n}=18$ for mean power analyses (corrected $P=0.0028$ ) and $\mathrm{n}=38$ for connectivity analyses (corrected $P=0.0013$ ). For each comparison, we reported both the $P$ value $<0.01$ and the $P$ value adjusted for multiple comparisons, in case the Bonferroni correction is too stringent since the cortical electrical activities are interactive, not totally independent.

\section{RESULTS}

The DRD4 exon III VNTR genotypes of the participants included 4-repeat/4-repeat $(N=129), \quad$ 2-repeat/4-repeat $(N=70), 2$-repeat/2-repeat $(N=16)$, other $<=4 /<=4(N=5)$ and $<=4 />4(N=13)$. In accordance with previous reports of HanChinese population, allele 4-repeat was predominant and the frequency of allele length $>4$ is low $[49,50]$, with the genotypes 4-repeat/5-repeat $(N=6), 4$-repeat/6-repeat $(N=6)$, 4-repeat/7-repeat $(N=1)$. No significant difference was noticed in the regional analyses of mean power. The connectivity analyses substantiated by TFCMI which combined Morlet wavelet transformation and mutual information analysis, revealed a relatively right-lateralized network with significant between-group differences. The significant connections comprised of homologous (inferior prefrontal F7-F8 and parietal C3-C4), fronto-temporal (F4-T4), fronto-parietal (F4-C4) and temporo-parietal (C4-T6) connections. Without

Table 1. Comparison of the Values of Mutual Information in DRD4 <=4-Repeat/<=4-Repeat Carriers (Upper Row) and Long Allele Carriers (>4-Repeat, Lower Row) for each EEG Connection-Frequency Pair

\begin{tabular}{|c|c|c|c|c|c|}
\hline & theta & alpha & beta & beta1 & beta2 \\
\hline \multirow[t]{2}{*}{ F7-F8 } & $22.1(4.0)$ & $30.2(12.4)$ & $14.1(6.5)$ & $15.4(7.6)$ & $10.6(2.2)$ \\
\hline & $22.2(4.2)$ & $29.6(14.5)$ & $13.3(5.5)$ & $14.7(7.5)$ & $9.6(1.0)$ \\
\hline \multirow[t]{2}{*}{$\mathrm{C} 3-\mathrm{C} 4$} & $21.4(4.2)$ & $26.2(11.4)$ & $13.2(4.8)^{*}$ & $13.7(5.1)$ & $10.4(2.2)$ \\
\hline & $21.6(4.2)$ & $20.8(5.7)$ & $11.8(0.9)$ & $12.9(1.1)$ & $9.9(1.1)$ \\
\hline \multirow[t]{2}{*}{ F4-T4 } & $22.8(4.9)$ & $25.5(10.6)$ & $14.1(5.4)$ & $15.0(5.6)$ & $11.2(2.9) *$ \\
\hline & $21.8(2.9)$ & $22.4(9.4)$ & $12.5(1.9)$ & $13.4(2.5)$ & $10.0(0.8)$ \\
\hline \multirow[t]{2}{*}{$\mathrm{F} 4-\mathrm{C} 4$} & $27.3(9.4)$ & $41.6(19.0)$ & $21.8(9.5)^{*}$ & $23.2(10.5)^{*}$ & $16.0(5.8)$ \\
\hline & $26.6(9.4)$ & $30.2(12.8)$ & $16.3(4.0)$ & $17.9(4.0)$ & $13.1(3.2)$ \\
\hline \multirow[t]{2}{*}{ T6-C4 } & $22.0(4.5)$ & $25.8(9.9)$ & $13.5(4.5)$ & $13.9(4.7)$ & $10.6(2.0)$ \\
\hline & $21.9(4.1)$ & $22.7(7.5)$ & $11.6(1.7)$ & $12.8(2.3)$ & $10.2(1.3)$ \\
\hline
\end{tabular}

The value of mutual information (x100) was expressed as "mean (STD)".

The between group differences with $P<0.01$ were marked in bold (two-sided)

The threshold of $P$ value after Bonferroni correction is $0.0013 ;{ }^{*} P<0.0013$ 
exception, the group 1 had higher connectivity strengths than group 2 at all the connections showing statistical significance, with a summarized topography illustrated in Fig. (1). The significant differences were aggregated at alpha and beta frequency bands. The detailed values and statistics of regional mean power over 18 electrodes and of mutual information over 38 channel-pairs across 5 frequency bands were respectively summarized in Supplementary Material Table S1 and Table S2 (http://www.websdj.idv.tw/kiki/rEEG_ DRD4.pdf); this article only reported the significant results, see Table 1.

We performed non-parametric analyses to examine the genetic effect on the global trend of brain dynamics. The long-allele DRD4 carriers had smaller regional mean power in 55 out of 90 electrode-frequency couples $(P=0.0132)$, and had reduced connectivity strength in 142 out of 190 connection-frequency pairs $\left(P=2.757 \times 10^{-12}\right)$.

We made further comparison of the subgroups of 4repeat/4-repeat and 2-repeat/4-repeat that have largest subject numbers. No regional power and no inter-regional connectivity strength revealed significant between group differences $(P<0.01$; data not shown). The negative findings justified our stratification strategy of lumping 2-repeat and 4repeat alleles together.

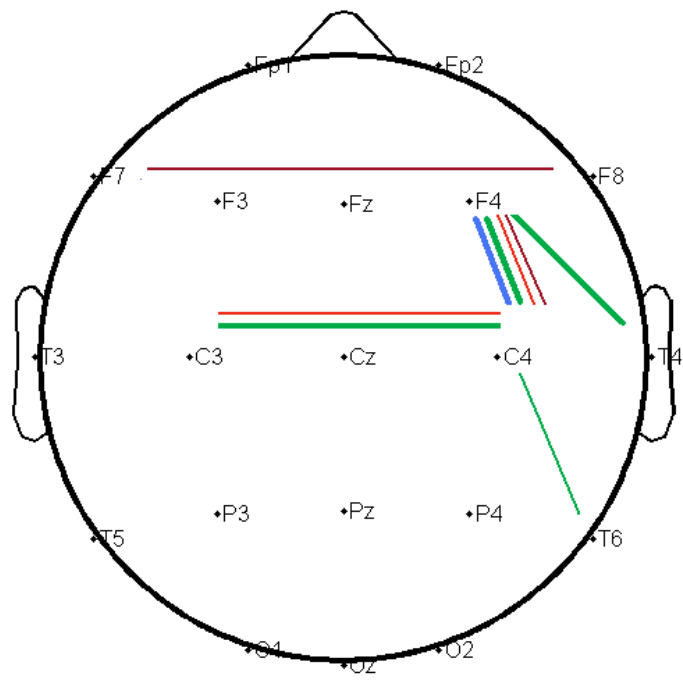

beta

Fig. (1). Summarized topography of independent t-test comparing $D R D 4$ VNTR polymorphism $<=4$-repeat $/<=4$-repeat carriers and $>4$-repeat carriers; cortical connections where the $P$ value less than 0.01 were shown in thin lines, while the $P$ value less than 0.0013 were shown in thick lines.

\section{DISCUSSION}

Dopamine receptor D4, DRD4, has been of interest in attention-deficit hyperactivity disorder [7, 8], and has been implicated in other neuropsychiatric conditions, such as drug abuse, Tourette syndrome and eating disorder and so on [10, $12,13,26-28,56]$. This study investigated the effects of DRD4 VNTR polymorphism at exon III on the local and the inter-regional neural profiles of resting EEG in healthy young females. We adopted an approach based on information theory, i.e. time-frequency cross mutual information (TFCMI) [46], to host non-linear relationship of neural interaction. The statistical comparisons were performed between two groups - with and without carriage of longrepeat allele, and the stratification strategy was conformed to previous literature of DRD4 VNTR polymorphism [7, 8, 1519]. Our regional power analysis did not show any significant differences, however, our connectivity analysis demonstrated that the long-allele carriers possessed decreased connection strengths compared with the short-allele $(<=4$ repeat $/<=4$-repeat) counterparts, aggregated at alpha and beta spectrum. The reduction in connectivity manifested itself as a global trend, most prominent at bi-frontal(inferior)/biparietal interactions and at a right-lateralized network, including fronto-temporal, fronto-parietal and parietotemporal connections.

The bio-psychological correlates bridging DRD4 VNTR polymorphism and the pathological state are still not clear. Although still debated, it was suggested that the long-allele was associated with risk taking, slower reaction time and lower persistence [15-17]. Notably, Congdon et al. adopted stop signal task as a proxy for impulsivity to investigate the influence of DRD4 VNTR on the capability of inhibitory control, and discovered that the long-allele carriers had longer stop signal reaction time, indicating greater difficulty in inhibiting a behavioral response to a stop signal [18]. In accord, Colzato et al. reported that the DRD4 7-repeat carriers had significantly higher scores on self-reported dysfunctional impulsivity [19]. The presence of a 7-repeat allele was associated with more errors of commission or omission in ADHD [20, 21]. Langley et al., reported that the ADHD children with 7-repeat allele had significantly more incorrect responses on the Matching Familiar Figures Test and shorter reaction time for incorrect responses on some neuropsychological tasks, indicating an inaccurate/impulsive response style [22]. The association of DRD4 long-allele and impulsivity is very intriguing given that impulsivity or disinhibition is a characteristic shared by many neuropsychiatric diseases relevant to DRD4, including ADHD, drug abuse, eating disorder and even Tourette syndrome (reviews) [26-28]. In addition, the relationship between inhibition/ impulsivity and DRD4 may partly explain the findings of previous studies that related DRD4 VNTR to lower persistence, risk taking and differences in reaction time [15-17]. The evidence linking dopamine system and impulsivity has also been endorsed by an acute administration of d-amphetamine [57]. In total, impulsivity or impairment in inhibitory control may be a common neuro-psychological mediator between DRD4 and various neuropsychiatric conditions. This interpretation is particularly interesting when coupled with our findings that the long-allele carriers have significant lower connectivity strengths, especially at inferior frontal gyri and over right hemisphere.

Right inferior frontal gyrus has been supposed to be a common hub for various neuropsychological tasks involving inhibitory operation across several response modalities [3744]. However, recent studies have suggested that left inferior frontal gyrus is also critical in performing response inhibition $[35,36]$. The impairment of response inhibition, a function linked to the inferior frontal gyri, was implicated in the manifestation of impulsive behaviors [40]. For long-allele carriers, our finding of reduced connectivity at bilateral inferior frontal gyrus might partly explain the noticed enhanced risk of disinhibition/impulsivity. The neural network relevant to response inhibition is not confined to right inferior frontal 
region but also comprises superior temporal gyrus and inferior parietal lobule, again with right-hemisphere dominance [58-60]. McNab et al., demonstrated that at a looser statistical threshold of analysis, response inhibition also engaged the right middle frontal gyrus and right parietal regions [39]. Together, the topography of our connectivity results at fronto-temporal and fronto-parietal connections is also compatible with the reported neural network of response inhibition beyond inferior frontal region. In addition, our main findings at the frontal and temporal areas are the cortical regions abundant in DRD4 mRNA expression $[2,3]$. The reduction in connectivity at bi-parietal interaction is out of our expectation. Nevertheless, a recent report by Heflin et al., demonstrated that bilateral parietal lobe atrophy best predicted poorer Stroop performance in 114 patients with cognitive impairment [61]. We thus regard that the reduced inter-regional interactions observed at the networks relevant to successful response inhibition may constitute neural mediators linking DRD4 VNTR long-allele and the diverse neuropsychiatric conditions with impulsivity/disinhibition as one of the characteristic attributes, such as ADHD, substance abuse, eating disorder and Tourette syndrome.

Another explanation for our finding of right-lateralized network could be relevant to the attention functioning associated with right hemisphere. The neuro-cognitive aberrations of ADHD were not restricted in motor response but also presented in perceptual/attentional domains [62-65]. A pattern of greater right-sided interference effects in ADHD children, in opposition to normal developing children, suggested a disruption in right hemisphere attentional networks in ADHD [62]. Correspondently, ADHD showed a significant bias in attention away from left space [64, 65]. It was reported that left-sided inattention (rightward bias) of ADHD predicted the treatment response to stimulants, which was further modulated by the dopamine system [66].

Although this study focused on frontal and temporal neocortices, it is noteworthy that the DRD4 also appeared in medial temporal structure $[2,3]$. In the basal nucleus of amygdala, the amounts of DRD4 mRNAs were significantly higher in the subjects with major depression [14]. The DRD4 VNTR polymorphism differentiated functional neural changes in mesocortico-limbic structures after exposure to alcohol cues, and in regions endorsing executive and somatosensory processes after exposure to smoking cues [67, 68]. It was reported that the DRD4 VNTR genotype also affected dopamine release in the ventral striatum following nicotine administration [69]. An animal study found that the modulatory effect of DRD4 on cerebral metabolism can be indirect, even on the structure that DRD4 was minimally expressed, such as the cerebellum [4]. Our results indicated that DRD4 VNTR polymorphism may have greater impact on interregional interaction than on regional neural activities. We thus encourage future studies to investigate the DRD4 influence on the neural interaction between cortical, subcortical and limbic structures to elucidate its modulatory effect at neural network level, which might correspond to the behavioral or symptomatic phenotypes of associated neuropsychiatric diseases.

We acknowledge that the above interpretation of impulsivity and attention is somewhat speculative. Nevertheless, the association of DRD4 VNTR polymorphism and inhibi- tion/impulsivity/risk-taking has been reported by several independent research groups $[15,16,18]$. In addition, DRD4 polymorphism affects wide repertoire of behavioral manifestation, including reward processing, response to novelty, cognitive control, attention, cortisol stress response, decision making, performance monitoring, parental responsivity, personality and temperament [17, 48, 70-80]. Each psychological entity may have its own correspondent gene-brain interaction. The attempt to bridge brain, gene and behavioral phenotypes is actually a very complicated issue and requires advanced multivariate models. We suggest future studies to incorporate comprehensive psychological, personality assessment and brain models to elucidate the complexity. Longitudinal studies are warranted to investigate whether the reduced connectivity strengths associated with DRD4 VNTR long allele enhance the risk of developing relevant psychiatric disorders. Since gonadal hormone may affect brain function and plasticity [81-83], as well as the integrity of dopamine system $[84,85]$, it is also desirable to explore the influence of different phases in menstrual cycle on genebrain interaction. Further studies are required to examine whether our results can be generalized to the male population.

\section{CONCLUSION}

The DRD4 gene has been linked to a variety of neurological and psychiatric conditions. There are few reports exploring the influence of DRD4 VNTR polymorphism at exon III on the regional neural activity and functional integration in the brain. Our analyses of resting EEG signals demonstrated that DRD4 VNTR long-allele was associated with reduced connectivity strengths at neural networks relevant to response inhibition. We suggest future DRD4 studies of imaging genetics incorporate connectivity analysis to unveil the genetic impact on cerebral network. Although the low frequency of long-repeat allele in DRD4 exon III VNTR in our sample (Han Chinese population) has been observed in other independent research samples [49, 50], replication of our results in a larger sample size or in other races with more balanced allele distribution is encouraged.

\section{CONFLICT OF INTEREST}

None declared.

\section{ACKNOWLEDGEMENT}

This work was supported by grant DOH94-NNB-1035 from the Department of Health, Taiwan, ROC and grant KS92-015 from the Kai-Suan Psychiatric HospitalKaohsiung. We are grateful to Mr. Higgins who helped prepare this manuscript.

\section{REFERENCES}

[1] Neve KA, Seamans JK, Trantham-Davidson H. Dopamine receptor signaling. J Recept Signal Transduct Res 2004; 24(3): 165-205.

[2] Meador-Woodruff JH, Grandy DK, Van Tol HH, et al. Dopamine receptor gene expression in the human medial temporal lobe. Neuropsychopharmacology 1994; 10(4): 239-48.

[3] Meador-Woodruff JH, Damask SP, Wang J, Haroutunian V, Davis $\mathrm{KL}$, Watson SJ. Dopamine receptor mRNA expression in human 
striatum and neocortex. Neuropsychopharmacology 1996; 15(1): 17-29.

[4] Michaelides M, Pascau J, Gispert JD, et al. Dopamine D4 receptors modulate brain metabolic activity in the prefrontal cortex and cerebellum at rest and in response to methylphenidate. Eur $\mathrm{J}$ Neurosci 2010; 32(4): 668-76.

[5] Van Tol HH, Wu CM, Guan HC, et al. Multiple dopamine D4 receptor variants in the human population. Nature 1992; 358(6382): 149-52.

[6] Asghari V, Sanyal S, Buchwaldt S, Paterson A, Jovanovic V, Van Tol HH. Modulation of intracellular cyclic AMP levels by different human dopamine D4 receptor variants. J Neurochem 1995; 65(3): 1157-65.

[7] Faraone SV, Doyle AE, Mick E, Biederman J. Meta-analysis of the association between the 7-repeat allele of the dopamine $\mathrm{D}(4)$ receptor gene and attention deficit hyperactivity disorder. Am J Psychiatry 2001; 158(7): 1052-7.

[8] Li D, Sham PC, Owen MJ, He L. Meta-analysis shows significant association between dopamine system genes and attention deficit hyperactivity disorder (ADHD). Hum Mol Genet 2006; 15(14): 2276-84.

[9] Kaplan AS, Levitan RD, Yilmaz Z, Davis C, Tharmalingam S, Kennedy JL. A DRD4/BDNF gene-gene interaction associated with maximum BMI in women with bulimia nervosa. Int $\mathrm{J}$ Eat Disord 2008; 41(1): 22-8.

[10] Dobashi I, Inada T, Hadano K. Alcoholism and gene polymorphisms related to central dopaminergic transmission in the Japanese population. Psychiatr Genet 1997; 7(2): 87-91.

[11] Haile CN, Kosten TR, Kosten TA. Pharmacogenetic treatments for drug addiction: cocaine, amphetamine and methamphetamine. Am J Drug Alcohol Abuse 2009; 35(3): 161-77.

[12] Mochi M, Cevoli S, Cortelli P, et al. A genetic association study of migraine with dopamine receptor 4 , dopamine transporter and dopamine-beta-hydroxylase genes. Neurol Sci 2003; 23(6): 301-5.

[13] Diaz-Anzaldua A, Joober R, Riviere JB, et al. Tourette syndrome and dopaminergic genes: a family-based association study in the French Canadian founder population. Mol Psychiatry 2004; 9(3): 272-7.

[14] Xiang L, Szebeni K, Szebeni A, et al. Dopamine receptor gene expression in human amygdaloid nuclei: elevated D4 receptor mRNA in major depression. Brain Res 2008; 1207: 214-24.

[15] Szekely A, Balota DA, Duchek JM, Nemoda Z, Vereczkei A, Sasvari-Szekely M. Genetic factors of reaction time performance: DRD4 7-repeat allele associated with slower responses. Genes Brain Behav 2011; 10(2): 129-36.

[16] Roussos P, Giakoumaki SG, Bitsios P. Cognitive and emotional processing in high novelty seeking associated with the L-DRD4 genotype. Neuropsychologia 2009; 47(7): 1654-9.

[17] Szekely A, Ronai Z, Nemoda Z, Kolmann G, Gervai J, SasvariSzekely M. Human personality dimensions of persistence and harm avoidance associated with DRD4 and 5-HTTLPR polymorphisms. Am J Med Genet B Neuropsychiatr Genet 2004; 126B(1): 106-10.

[18] Congdon E, Lesch KP, Canli T. Analysis of DRD4 and DAT polymorphisms and behavioral inhibition in healthy adults: implications for impulsivity. Am J Med Genet B Neuropsychiatr Genet 2008; 147B(1): 27-32.

[19] Colzato LS, van den Wildenberg WP, Van der Does AJ, Hommel B. Genetic markers of striatal dopamine predict individual differences in dysfunctional, but not functional impulsivity. Neuroscience 2010; 170(3): 782-8.

[20] Kieling C, Roman T, Doyle AE, Hutz MH, Rohde LA. Association between DRD4 gene and performance of children with ADHD in a test of sustained attention. Biol Psychiatry 2006; 60(10): 1163-5.

[21] Johnson KA, Kelly SP, Robertson IH, et al. Absence of the 7repeat variant of the DRD4 VNTR is associated with drifting sustained attention in children with ADHD but not in controls. Am J Med Genet B Neuropsychiatr Genet 2008; 147B(6): 927-37.

[22] Langley K, Marshall L, van den Bree M, et al. Association of the dopamine D4 receptor gene 7-repeat allele with neuropsychological test performance of children with ADHD. Am J Psychiatry 2004; 161(1): 133-8

[23] Strobel A, Debener S, Anacker K, Muller J, Lesch KP, Brocke B. Dopamine D4 receptor exon III genotype influence on the auditory evoked novelty P3. Neuroreport 2004; 15(15): 2411-5.
[24] Demiralp T, Herrmann CS, Erdal ME, et al. DRD4 and DAT1 polymorphisms modulate human gamma band responses. Cereb Cortex 2007; 17(5): 1007-19.

[25] Kramer UM, Rojo N, Schule R, et al. ADHD candidate gene (DRD4 exon III) affects inhibitory control in a healthy sample. BMC Neurosci 2009; 10: 150 .

[26] Waxman SE. A systematic review of impulsivity in eating disorders. Eur Eat Disord Rev 2009; 17(6): 408-25.

[27] Sheppard DM, Bradshaw JL, Purcell R, Pantelis C. Tourette's and comorbid syndromes: obsessive compulsive and attention deficit hyperactivity disorder. A common etiology? Clin Psychol Rev 1999; 19(5): 531-52.

[28] Koob GF, Volkow ND. Neurocircuitry of addiction. Neuropsychopharmacology 2010; 35(1): 217-38.

[29] Mulert C, Juckel G, Brunnmeier M, et al. Prediction of treatment response in major depression: integration of concepts. J Affect Disord 2007; 98(3): 215-25.

[30] Jelic V, Kowalski J. Evidence-based evaluation of diagnostic accuracy of resting EEG in dementia and mild cognitive impairment. Clin EEG Neurosci 2009; 40(2): 129-42.

[31] Neuper C, Grabner RH, Fink A, Neubauer AC. Long-term stability and consistency of EEG event-related (de-)synchronization across different cognitive tasks. Clin Neurophysiol 2005; 116(7): 1681-94.

[32] Hermens DF, Soei EX, Clarke SD, Kohn MR, Gordon E, Williams LM. Resting EEG theta activity predicts cognitive performance in attention-deficit hyperactivity disorder. Pediatr Neurol 2005; 32(4): 248-56.

[33] Hoptman MJ, Davidson RJ. Baseline EEG asymmetries and performance on neuropsychological tasks. Neuropsychologia 1998 36(12): 1343-53.

[34] Coben LA, Chi D, Snyder AZ, Storandt M. Replication of a study of frequency analysis of the resting awake EEG in mild probable Alzheimer's disease. Electroencephalogr Clin Neurophysiol 1990; 75(3): 148-54.

[35] Matsubara M, Yamaguchi S, Xu J, Kobayashi S. Neural correlates for the suppression of habitual behavior: a functional MRI study. J Cogn Neurosci 2004; 16(6): 944-54.

[36] Swick D, Ashley V, Turken AU. Left inferior frontal gyrus is critical for response inhibition. BMC Neurosci 2008; 9: 102.

[37] Kenner NM, Mumford JA, Hommer RE, Skup M, Leibenluft E, Poldrack RA. Inhibitory motor control in response stopping and response switching. J Neurosci 2010; 30(25): 8512-8.

[38] Hampshire A, Chamberlain SR, Monti MM, Duncan J, Owen AM. The role of the right inferior frontal gyrus: inhibition and attentional control. Neuroimage 2010; 50(3): 1313-9.

[39] McNab F, Leroux G, Strand F, Thorell L, Bergman S, Klingberg T. Common and unique components of inhibition and working memory: an fMRI, within-subjects investigation. Neuropsychologia 2008; 46(11): 2668-82.

[40] Chamberlain SR, Sahakian BJ. The neuropsychiatry of impulsivity. Curr Opin Psychiatry 2007; 20(3): 255-61.

[41] Chikazoe J, Konishi S, Asari T, Jimura K, Miyashita Y. Activation of right inferior frontal gyrus during response inhibition across response modalities. J Cogn Neurosci 2007; 19(1): 69-80.

[42] Aron AR, Robbins TW, Poldrack RA. Inhibition and the right inferior frontal cortex. Trends Cogn Sci 2004; 8(4): 170-7.

[43] Konishi S, Nakajima K, Uchida I, Sekihara K, Miyashita Y. No-go dominant brain activity in human inferior prefrontal cortex revealed by functional magnetic resonance imaging. Eur J Neurosci 1998; 10(3): 1209-13.

[44] Konishi S, Nakajima K, Uchida I, Kikyo H, Kameyama M, Miyashita Y. Common inhibitory mechanism in human inferior prefrontal cortex revealed by event-related functional MRI. Brain 1999; 122 (Pt 5): 981-91.

[45] Nagano-Saito A, Leyton M, Monchi O, Goldberg YK, He Y, Dagher A. Dopamine depletion impairs frontostriatal functional connectivity during a set-shifting task. J Neurosci 2008; 28(14): 3697-706.

[46] Chen CC, Hsieh JC, Wu YZ, et al. Mutual-information-based approach for neural connectivity during self-paced finger lifting task. Hum Brain Mapp 2008; 29(3): 265-80.

[47] Muller J, Dreisbach G, Brocke B, Lesch KP, Strobel A, Goschke T. Dopamine and cognitive control: the influence of spontaneous eyeblink rate, DRD4 exon III polymorphism and gender on flexibility in set-shifting. Brain Res 2007; 1131(1): 155-62. 
[48] Kang JI, Namkoong K, Kim SJ. Association of DRD4 and COMT polymorphisms with anger and forgiveness traits in healthy volunteers. Neurosci Lett 2008; 430(3): 252-7.

[49] Wang E, Ding YC, Flodman P, et al. The genetic architecture of selection at the human dopamine receptor D4 (DRD4) gene locus. Am J Hum Genet 2004; 74(5): 931-44.

[50] Tsai SJ, Yu YW, Chen TJ, Chen MC, Hong CJ. Association analysis for dopamine D3 receptor, dopamine D4 receptor and dopamine transporter genetic polymorphisms and P300 eventrelated potentials for normal young females. Psychiatr Genet 2003; 13(1): 51-3.

[51] Duffy FH, Lyer G, Surwillo WW. Clinical electroencephalography and topographical brain mapping. New York: Springer-Verlag 1989.

[52] Lee TW, Yu YW, Hong CJ, Tsa SJ, Wu HC, Chen TJ. The effects of Catechol-O-methyl transferase polymorphism Val158Met on functional connectivity in healthy young females: a resting EEG study. Brain Res 2011; 1377: 21-31.

[53] Fingelkurts AA, Rytsala H, Suominen K, Isometsa E, Kahkonen S. Impaired functional connectivity at EEG alpha and theta frequency bands in major depression. Hum Brain Mapp 2007; 28(3): 247-61.

[54] Shaikh S, Collier D, Kerwin RW, et al. Dopamine D4 receptor subtypes and response to clozapine. Lancet 1993; 341(8837): 116.

[55] Hong CJ, Lee YL, Sim CB, Hwu HG. Dopamine D4 receptor variants in Chinese sporadic and familial schizophrenics. Am J Med Genet 1997; 74(4): 412-5.

[56] Chen $\mathrm{CK}, \mathrm{Hu} \mathrm{X}$, Lin SK, et al. Association analysis of dopamine D2-like receptor genes and methamphetamine abuse. Psychiatr Genet 2004; 14(4): 223-6.

[57] de Wit H, Enggasser JL, Richards JB. Acute administration of damphetamine decreases impulsivity in healthy volunteers. Neuropsychopharmacology 2002; 27(5): 813-25.

[58] Horn NR, Dolan M, Elliott R, Deakin JF, Woodruff PW. Response inhibition and impulsivity: an fMRI study. Neuropsychologia 2003; 41(14): 1959-66.

[59] Garavan H, Ross TJ, Stein EA. Right hemispheric dominance of inhibitory control: an event-related functional MRI study. Proc Natl Acad Sci USA 1999; 96(14): 8301-6.

[60] Forstmann BU, Jahfari S, Scholte HS, Wolfensteller U, van den Wildenberg WP, Ridderinkhof KR. Function and structure of the right inferior frontal cortex predict individual differences in response inhibition: a model-based approach. J Neurosci 2008; 28(39): 9790-6.

[61] Heflin LH, Laluz V, Jang J, Ketelle R, Miller BL, Kramer JH. Let's inhibit our excitement: The relationships between Stroop, behavioral disinhibition, and the frontal lobes. Neuropsychology 2011; 25(5): 655-65.

[62] Chan E, Mattingley JB, Huang-Pollock C, et al. Abnormal spatial asymmetry of selective attention in ADHD. J Child Psychol Psychiatry 2009; 50(9): 1064-72.

[63] Rolfe MH, Hamm JP, Waldie KE. Differences in paper-and-pencil versus computerized line bisection according to ADHD subtype and hand-use. Brain Cogn 2008; 66(2): 188-95.

[64] Dobler VB, Anker S, Gilmore J, Robertson IH, Atkinson J, Manly T. Asymmetric deterioration of spatial awareness with diminishing levels of alertness in normal children and children with ADHD. J Child Psychol Psychiatry 2005; 46(11): 1230-48.

[65] George M, Dobler V, Nicholls E, Manly T. Spatial awareness, alertness, and ADHD: the re-emergence of unilateral neglect with time-on-task. Brain Cogn 2005; 57(3): 264-75.

[66] Bellgrove MA, Barry E, Johnson KA, et al. Spatial attentional bias as a marker of genetic risk, symptom severity, and stimulant response in ADHD. Neuropsychopharmacology 2008; 33(10): 2536-45.
[67] Filbey FM, Ray L, Smolen A, Claus ED, Audette A, Hutchison KE. Differential neural response to alcohol priming and alcohol taste cues is associated with DRD4 VNTR and OPRM1 genotypes. Alcohol Clin Exp Res 2008; 32(7): 1113-23.

[68] McClernon FJ, Hutchison KE, Rose JE, Kozink RV. DRD4 VNTR polymorphism is associated with transient fMRI-BOLD responses to smoking cues. Psychopharmacology 2007; 194(4): 433-41.

[69] Brody AL, Mandelkern MA, Olmstead RE, et al. Gene variants of brain dopamine pathways and smoking-induced dopamine release in the ventral caudate/nucleus accumbens. Arch Gen Psychiatry 2006; 63(7): 808-16.

[70] Schmidt LA, Fox NA, Perez-Edgar K, Hamer DH. Linking gene, brain, and behavior: DRD4, frontal asymmetry, and temperament. Psychol Sci 2009; 20(7): 831-7.

[71] Williams LM, Gatt JM, Hatch A, et al. The integrate model of emotion, thinking and self regulation: an application to the "paradox of aging". J Integr Neurosci 2008; 7(3): 367-404.

[72] Holmboe K, Nemoda Z, Fearon RM, Csibra G, Sasvari-Szekely M, Johnson MH. Polymorphisms in dopamine system genes are associated with individual differences in attention in infancy. Dev Psychol 2010; 46(2): 404-16.

[73] Armbruster D, Mueller A, Moser DA, Lesch KP, Brocke B, Kirschbaum C. Interaction effect of D4 dopamine receptor gene and serotonin transporter promoter polymorphism on the cortisol stress response. Behav Neurosci 2009; 123(6): 1288-95.

[74] Ha RY, Namkoong K, Kang JI, Kim YT, Kim SJ. Interaction between serotonin transporter promoter and dopamine receptor D4 polymorphisms on decision making. Prog Neuropsychopharmacol Biol Psychiatry 2009; 33(7): 1217-22.

[75] Kramer UM, Cunillera T, Camara E, et al. The impact of catecholO-methyltransferase and dopamine D4 receptor genotypes on neurophysiological markers of performance monitoring. J Neurosci 2007; 27(51): 14190-8.

[76] Golimbet VE, Alfimova MV, Gritsenko IK, Ebstein RP. Relationship between dopamine system genes and extraversion and novelty seeking. Neurosci Behav Physiol 2007; 37(6): 601-6.

[77] Dreisbach G, Muller J, Goschke T, et al. Dopamine and cognitive control: the influence of spontaneous eyeblink rate and dopamine gene polymorphisms on perseveration and distractibility. Behav Neurosci 2005; 119(2): 483-90.

[78] Fan J, Fossella J, Sommer T, Wu Y, Posner MI. Mapping the genetic variation of executive attention onto brain activity. Proc Natl Acad Sci USA 2003; 100(12): 7406-11.

[79] Strobel A, Lesch KP, Jatzke S, Paetzold F, Brocke B. Further evidence for a modulation of Novelty Seeking by DRD4 exon III 5-HTTLPR, and COMT val/met variants. Mol Psychiatry 2003; 8(4): 371-2.

[80] Fossella J, Sommer T, Fan J, et al. Assessing the molecular genetics of attention networks. BMC Neurosci 2002; 3: 14.

[81] Bayer U, Hausmann M. Sex hormone therapy and functional brain plasticity in postmenopausal women. Neuroscience 2011; 191: 118 28.

[82] Tobet S, Knoll JG, Hartshorn C, et al. Brain sex differences and hormone influences: a moving experience? J Neuroendocrinol 2009; 21(4): 387-92.

[83] Lee AW, Pfaff DW. Hormone effects on specific and global brain functions. J Physiol Sci 2008; 58(4): 213-20.

[84] Leranth C, Roth RH, Elsworth JD, Naftolin F, Horvath TL, Redmond DE Jr. Estrogen is essential for maintaining nigrostriatal dopamine neurons in primates: implications for Parkinson's disease and memory. J Neurosci 2000; 20(23): 8604-9.

[85] Hruska RE, Ludmer LM, Pitman KT, De Ryck M, Silbergeld EK. Effects of Estrogen on Striatal Dopamine receptor function in male and female rats. Pharmacol Biochem Behav 1982;16(2): 285-91.

(C) Lee et al.; Licensee Bentham Open.

This is an open access article licensed under the terms of the Creative Commons Attribution Non-Commercial License (http://creativecommons.org/licenses/by$\mathrm{nc} / 3.0 /$ ), which permits unrestricted, non-commercial use, distribution and reproduction in any medium, provided the work is properly cited. 